\title{
Effect of an isoflavones-containing red clover preparation and alkaline supplementation on bone metabolism in ovariectomized rats
}

\author{
S Kawakita' \\ F Marotta ${ }^{2}$ \\ Y Naito ${ }^{3}$ \\ U Gumaste ${ }^{4}$ \\ $S$ Jain ${ }^{5}$ \\ J Tsuchiya' \\ E Minelli²
}

'Biokenkyusho Research Laboratory, Shizuoka, Japan; ${ }^{2} \mathrm{WHO}$-cntr for Biotechnology and Natural Medicine, University of Milan, Milan, Italy; ${ }^{3}$ Immunology Research Institute and Clinic, Nagoya, Japan; ${ }^{4}$ Agharkar Research Institute, Pune, Maharashtra, India; ${ }^{5}$ Department of Food Science and Human Nutrition, University of Illinois, Urbana-Champaign, IL, USA

Correspondence: $\mathrm{F}$ Marotta Piazza Firenze, 12, 20154 Milano, Italy

Tel +39024077243

Fax +39024077243

Email fmarchimede@libero.it
Abstract: The aim of this study was to test the combined effect of a quality-controlled red clover extract (RCE) standardized to contain 40\% isoflavones by weight (genistein, daidzein, biochanin A, and formononetin present as hydrolyzed aglycones) together with a modified alkaline supplementation on bone metabolic and biomechanical parameters in an experimental model of surgically-induced menopause. Sprague-Dawley female rats were maintained under controlled standard conditions of light and fed with conventional food of standard calcium content and no alfalfa or soybean components. Rats were randomized into four groups: Group A represented normal rats (sham operated) while three other groups were ovariectomized (OVX) and fed for three months as follows: standard food (group B), $6 \mathrm{mg} / \mathrm{kg} / \mathrm{day}$ food mixed with RCE (Group C), or given $6 \mathrm{mg} / \mathrm{kg}$ /day of RCE plus a modified alkaline supplementation (BP) through a nasogastric tube at a dose of $16 \mathrm{mg}$ (group D). The animals were killed 90 days after surgery. As compared to group B, RCE or RCE + BP treatments brought about significantly higher level of estradiol and mitigated the weight loss of the uterus and improved maximum load of the femoral neck. Osteocalcin level showed an over $65 \%$ increase in group B but both RCE and $\mathrm{RCE}+\mathrm{BP}$ treatments prevented such abnormality with a significantly better result in RCE + BP group which virtually normalized such parameter as well as urinary excretion of DPD. Group C and $\mathrm{D}$ reduced the over $20 \%$ loss of bone mineral density and bone mineral content/body weight ratio observed in untreated post-ovariectomy group. Untreated ovariectomy caused about $48 \%$ decrease of cancellous bone mass in the femoral neck while this abnormality was prevented at similar extent by both RCE and RCE + BP treatments. Ovariectomy determined an over 80\% increase of bone alkaline phosphatase (BALP) level but both RCE and RCE + BP treatments significantly mitigated such variable. The BALP decrease yielded by the combined RCE + BP treatment was statistically lower than RCE alone. Taken together these data show that red clover preparation in dosages amenable to clinical practice do improve OVX-induced osteoporosis while a mild metabolic alkalosis might further synergize some therapeutic aspects.

Keywords: red clover, alkaline supplementation, osteoporosis, ovariectomy

\section{Introduction}

Osteoporosis represents the most common metabolic bone disease ${ }^{1}$ and WHO defines it as a systematic skeletal disease characterized by low bone mass and micro-architectural deterioration of bone tissue, with a consequent increase in bone fragility and susceptibility to fractures. ${ }^{2}$ The most common type of osteoporosis is the bone loss associated with menopausal ovarian hormone deficiency. The incidence of osteoporosis is increasing worldwide as populations age and women are generally disproportionably affected by osteoporosis in developed countries and four times more likely than men to develop osteoporosis. Moreover, fracture rates among women are approximately twice as high as men. ${ }^{3}$ Indeed, estrogen deficiency is regarded as a critical cause of osteoporosis, which can result from naturally or surgically induced menopause and endocrine 
disorders that reduce estrogen secretion in premenopausal women. It influences osteoclast which enhances bone loss by stimulating bone resorption. Convention therapies for treating osteoporosis in women include hormone replacement therapy, either estrogen alone or in combination with progesterone, estrogen receptors-modulators, calcitonin and bisphosphonates. $^{4}$

The Women's Health Initiative initial study results demonstrated a statistically significant reduction in fractures, including hip fractures, in a large group of otherwise healthy women using hormone therapy. However, it also poses a number of cautions ${ }^{5}$ thus prompting active research to identify new agents for the treatment of postmenopausal osteoporosis with minimal side effects. Of all the natural alternatives currently under investigation, phytoestrogens appear to offer the most potential for the prevention of bone loss and they have attracted new attention as a possible agent to prevent and treat postmenopausal osteoporosis in women. From an epidemiological standpoint, Asian female population have lower rates of osteoporosis-related fractures than Western women ${ }^{6}$ and, besides a likely different genomic profile and different hip axis length, this is suspected to be in part because they consume more soy products such as miso and tofu that are rich in isoflavones, than Western women do. ${ }^{7,8}$ The evidence from both experimental in vivo and human studies have confirmed that soy isoflavones favorably impact bone health. ${ }^{9}$

Although the potential beneficial effects of a high phytoestrogen diet have been reported in some articles, ${ }^{10,11}$ only few articles are available on the potential impact of these compounds on osteoporosis treatment and/or prevention. The preventive effect of soybean protein on ovariectomized (OVX)-induced bone loss ${ }^{12}$ has been attributed to its high content of the isoflavones genistein and daidzein phytoestrogens which possess a range of biological effects including estrogenic, antiestrogenic, antiviral and antiproliferative actions. $^{13}$

Red clover (Trifolium pratense L, Fabaceae) botanical dietary supplements have received much attention recently for their potential use in the treatment of menopause symptoms, maintenance/improvement cardiovascular health and for the reported benign effects on the breast, endometrium and neural structure besides for its safety. ${ }^{14-17}$ As for the bonepreserving property, the effects of red clover have not been examined as extensively as for soy. Only three randomized controlled trials of red clover isoflavones for bone loss have been published but with two of them clearly demonstrating a positive effect on bone mineral density (BMD) ${ }^{18,19}$ as well as an increase in bone formation markers.
The ovariectomized rat is the most appropriate model for studying the mechanism as well as potential treatments of postmenopausal osteoporosis in humans. It is also a good model to study the efficacy of a number of candidates for their prevention and/or reversal of bone loss. ${ }^{20}$ For instance, Dai and colleagues ${ }^{21}$ have very recently shown that genistein preserved the biomechanical quality of the vertebral trabecular bone regardless of the microstructure and BMD in ovariectomized rats although such experimental data have to be interpreted with caution in terms of humans applicability. Besides the important endocrine modulator of bone matrix, it has been shown that supplementation with alkaline salts have significant effects on markers of bone turnover and urinary $\mathrm{pH}$ and net acid excretion. ${ }^{22-24}$ We have recently demonstrated that a balanced alkaline supplementation could significantly prevent high protein-induced chronic acidosis with hypercalciuria and hyperphosphaturia ${ }^{25}$ while also improving muscular metabolism under exhaustive exercise. ${ }^{26}$ Accordingly, animal and in vitro studies have clearly elucidated the mechanisms for the effect of metabolic acidosis on bone metabolism ${ }^{27,28}$ and have also established that metabolic acidosis is responsible for the effect of modified $\mathrm{pH}$ on bone cells and that this effect is mediated by prostaglandin $\mathrm{E}_{2} \cdot{ }^{29,30}$

Thus, the aim of this study was to test the combined effect of a quality-controlled red clover extract (RCE) standardized to contain $40 \%$ isoflavones by weight (genistein, daidzein, biochanin A, and formononetin present as hydrolyzed aglycones) together with a modified alkaline supplementation on bone metabolic and biomechanical parameters in an experimental model of surgically-induced menopause.

\section{Materials and methods}

\section{Study protocol}

Ninety-day-old Sprague-Dawley nulliparous female rats, with mean weight of $210 \mathrm{~g}$, were selected and were acclimatized for 2 weeks under controlled standard conditions of light $(12 / 24 \mathrm{~h})$ and temperature $\left(26 \pm 1^{\circ} \mathrm{C}\right)$. Food pellets (without any alfalfa or soybean components and with standard $0.44 \%$ calcium content which was slightly reduced in treated groups so to compensate with the calcium contained in the alkaline supplementation) and tap water were provided ad libitum. For experimental purposes animals fasted overnight but were allowed free access to water. All animal procedures were performed according to approved protocols and in accordance with the recommendations for the proper care and use of laboratory animals. Eighty rats were randomized into four groups of 20 animals each. Group A represented normal rats (sham operated) while three other groups underwent bilateral 
ovariectomy (OVX) under isoflurane anaesthesia and fed from the following day as follows. One OVX group was kept on standard food (group B) while the remaining two OVX groups of rats were started on a different daily feeding for 3 months as follows: Group C was fed with $6 \mathrm{mg} / \mathrm{kg} /$ day food mixed with RCE (Menoflavon Forte ${ }^{\circledR}$, Named, Lesmo, Italy). This dose was chosen on the basis of prior in-house dose-response test and aiming to be not too different from the one commonly used in clinics (240 mg/day). Group D was given $6 \mathrm{mg} / \mathrm{kg} /$ day of RCE plus a modified alkaline supplementation through a nasogastric tube at a dose of $16 \mathrm{mg}$ (BP: calcium bicarbonate, sodium bicarbonate, magnesium and potassium carbonate, and bisodium phosphate). At the end of the experimental period, the animals were fast overnight, and sacrificed by cervical dislocation while the blood being collected from the carotid artery. The serum obtained by centrifugal separation was stored at $-30{ }^{\circ} \mathrm{C}$ until assay. The animals were killed 90 days after surgery and the femurs were removed to carry out the below-mentioned studies.

\section{Serum estradiol and osteocalcin}

Serum estradiol was assayed by a direct radioimmunoassay kit (n. 0355, Hindawi-Tech, Bangalore, India) The antibody used was \#244 anti-estradiol-6-BSA serum at a dilution of $1: 3,000$. Intra- and interassay CV were $12 \%$ and $11 \%$, respectively.

Serum osteocalcin was determined by a species-specific ${ }^{31}$ RIA kit with rat ${ }^{125} \mathrm{I}$-labeled OC, goat anti-rat osteocalcin antibody (Biochemical Technologies, Stoughton, MA, USA). using a gamma counter (Hewlett-Packard, Palo Alto, CA, USA) where the analytic kit recognizes also the N-terminal midfragment thus allowing the correction of potential degradation of the intact molecule. The intra- and interassay coefficients of variation were below $4.5 \%$ and $7 \%$, respectively, and the sensitivity is $0.54 \mathrm{ng} / \mathrm{mL}$.

\section{Determination of uterine weight}

At necropsy, the uteri were collected, trimmed of fat and connective tissue, cut open and drained of intrauterine fluid, weighed, frozen in $1.5-\mathrm{mL}$ cryogenic vials on dry ice, and stored at $-70{ }^{\circ} \mathrm{C}$ until analysis.

\section{Bone mineral and cancellous bone mass assessment}

Bone densitometry of the whole femur was performed with Dual-Energy X-Ray Absorption (DEXA) scanner (GE-Lunar Corp., Madison, WI, USA) running software version 1.45 equipped with a high resolution for the evaluation of bone mineral in small subjects. Both bone mineral content (BMC) and BMD were determined, the former calculated as absolute value and in relationship with body mass too. The coefficient of variation for total BMD as measured in prior in-house tests is $1.0 \% \pm 0.3 \%$ and scan acquisition time was $5 \mathrm{~min} /$ animal

For cancellous bone mass analysis, the proximal femur was sawed off, stained with Villaneuva stain, defatted and embedded undecalcified in methyl methacrylate. Longitudinal bone sectioning was then started at approximately one third the depth of the femoral neck with a microtome set at $4 \mu \mathrm{m}$ thickness sections. The percentage of cancellous bone mass (BV/TV) was measured with a computer-linked light microscope from the entire cancellous spongiosa of the proximal femur in a standardized mid region $1 \mathrm{~mm}$ distal to the growth plate--metaphyseal junction.

\section{Measurement of bone mechanics}

Bones were kept in $\mathrm{NaCl}(9 \mathrm{~g} / \mathrm{l})$ at $4{ }^{\circ} \mathrm{C}$ until whole left femoral breaking force was determined $24 \mathrm{~h}$ afterwards with three-point loading. Prior to mechanical testing, the left femurs were slightly thawed and held at room temperature on the day of test, then connective tissues were carefully removed, the length of the femurs was measured with a micrometer. The load was applied perpendicularly to the long axis of the femur in the mid-length of the bone supported on its epiphyses. The load increased at a rate of $100 \mathrm{~N} / \mathrm{min}$. The load was measured by the sensor with a strain gauge; the deformation was measured by an inductive sensor. The signals sent by the sensors were amplified and registered. The load-deformation curves, obtained for each bone, representing the relationships between load applied to the bone and deformation in response to the load, were analyzed. The load-deformation curve can be divided into the elastic deformation region and the plastic deformation region. Within the elastic deformation region, the slope of the linear part of the load-deformation curve, representing the extrinsic stiffness of bone, was tested. Within the plastic deformation region, the ultimate load and the breaking load were determined. The ultimate load is the maximum load sustained by the bone. The breaking load is the load at which the bone actually breaks. The force and energy (area under the curve, $\mathrm{N} 3 \mathrm{~mm}$ ) necessary for the break at the center of the femur shaft were measured. Deformation caused by the applied loads was also measured. The mechanical strength of the left femoral neck was determined using a compression test with the same instrument. In femur shaft mechanical test, all femoral specimens were broken into two pieces, the proximal part was used and embedded in resin up to the lesser trochanter and a cylinder was shaped. The sample was inserted 
into a steel support in a vertical orientation. The mechanical resistance to failure was tested by applying a vertical load on the femoral head at a speed of $2 \mathrm{~mm} / \mathrm{min}$. All mechanic tests data were expressed as Newton $(\mathrm{N})$.

\section{Total (TALP) and bone-specific (BALP) alkaline phosphatase and urinary deoxypyridinoline assay}

Total alkaline phosphatase (TALP) was measured spectrophotometrically on a using p-nitrophenyl phosphate as substrate. The intra-assay CV was $1.9 \%$ and the inter-assay CV $2.8 \%$ at a mean value of $482 \mu / 1$. For bone alkaline phosphatase (BALP) assay the samples were pretreated by incubating $300 \mu \mathrm{l}$ of serum with $30 \mu \mathrm{l}$ Triton $\mathrm{X}(20 \mathrm{~g} / \mathrm{l})$ for $30 \mathrm{~min}$ at $37^{\circ} \mathrm{C}$. An aqueous solution of wheat germ lectin (300 $\mu$ l Sigma-9640, $5 \mathrm{~g} / \mathrm{l}$ in distilled water) was then added, and the samples were mixed and incubated for $30 \mathrm{~min}$ at $37^{\circ} \mathrm{C}$. After centrifugation at $2000 \mathrm{~g}$ for $10 \mathrm{~min}$, the alkaline phospatase activity in the supernatant was determined as above, and calculated as the difference between total and supernatant activity after correction for sample dilution. This was measured spectrophotometrically in the supernatant after precipitation with wheat germ lectin. The intra-assay CV was 5.3\% and the inter-assay CV 6.1\% at a mean value of $240 \mu / 1$. Urinary deoxypyridinoline (DPD) excretion was determined by competitive radioimmunoassay with rat monoclonal anti-DPD antibody coated to the inner surface of a polystyrene tube and ${ }^{125}$ I-labeled DPD (DPD-uri kit, HindawiTech, Bangalore, India). The sensitivity was $2.5 \mathrm{nmol} / \mathrm{L}$. The intra- and interassay variations were $5 \%$ and $8 \%$, respectively. Results were expressed as nmol DPD/mmol creatinine.

\section{Statistical analysis}

All data were expressed as means \pm standard deviation. Statistical analysis was done using StatView J-4.5 (Abacus Concepts Inc., Berkeley, CA, USA). Significance of the results was determined by one-way analysis of variance (ANOVA) and Bonferroni analysis was applied to test the differences between individual groups. A $p$ value $<0.05$ was considered statistically significant.

\section{Results}

RCE and BP supplementation did not cause any overt side effects and the histology and mass of the internal organs examined (liver, kidney, spleen, pancreas and heart) were within normal limits (data not shown). Group B OVX rats showed an almost $45 \%$ gain of body mass increase as compared to sham-operated rats (group A) ( $\mathrm{p}<0.01$, data not shown) but this effect was significantly reduced to less than
$30 \%$ in both treatment groups ( $\mathrm{C}$ and $\mathrm{D}$ vs $\mathrm{B}, \mathrm{p}<0.05)$. During our experiment, no significant difference was found among the various groups, ovariectomy did not affect the femur and tibia length compared to the sham rats. Treatment with either RCE and RCE + BP did not influence the growth rates of these long bones (data not shown).

\section{Effects of ovariectomy, RCE, and RCE + BP supplementation on serum estradiol, osteocalcin levels, and uterine weight}

As compared to OVX group devoid of any specific supplement (group B), RCE or RCE + BP treatment brought about a comparably and significantly higher level of estradiol $(\mathrm{p}<0.05)$ although this resulted about 30\% lower than in sham-operated rats (group A) ( $p<0.05$, Figure 1A). Ovariectomy brought about a decrease of over $70 \%$ of the uterus weight $(\mathrm{p}<0.01)$. Such loss was significant less pronounced when OVX rats were given RCE or RCE + BP ( $p<0.05$ vs group B) although it was still about 50\% lower that in the sham-operated rats ( $\mathrm{p}<0.01$, Figure 1B). Osteocalcin level showed an over $65 \%$ increase in untreated OVX rats (group B) as compared to sham-operated ones (group A). Both RCE and RCE + BP treatments remarkably prevented such abnormality (C and D vs B, p $<0.01$, Figure $1 \mathrm{C}$ ) with a significantly better result in RCE + BP group which virtually normalized such parameter $(\geq 25 \%$ vs $C$, $p<0.05)$.

\section{Bone mineral and cancellous bone mass assessment}

Daily supplementation of OVX rats with RCE or RCE + BP reduced the over $20 \%$ loss of BMD observed in untreated post-ovariectomy group (C and D vs B, p $<0.05$, Figure $2 \mathrm{~A}$ ). Combined treatment with RCE and BP (group D) showed a trend better value ( $>9 \%$ vs group $C$ ) but this did not reach a statistical significance. BMC as absolute value was not affected by ovariectomy nor by treatments (data not shown). However, when calculated in relationship with body mass, it appeared a significant decrease of this parameters in rats which had underwent ovariectomy and fed standard food (group B) ( $p<0.05$, Figure 2B). Such abnormality was abolished by the concomitant treatment with either treatment employed ( $\mathrm{p}<0.05$ vs group B). Untreated ovariectomy (group B) caused about 48\% decrease of cancellous bone mass in the femoral neck as compared to sham-operated rats ( $\mathrm{p}<0.01$, Figure $2 \mathrm{C}$ ) while this abnormality was prevented at similar extent by both RCE and RCE + BP treatments $(\mathrm{p}<0.05$ vs group B). 
A

\section{Estradiol level}

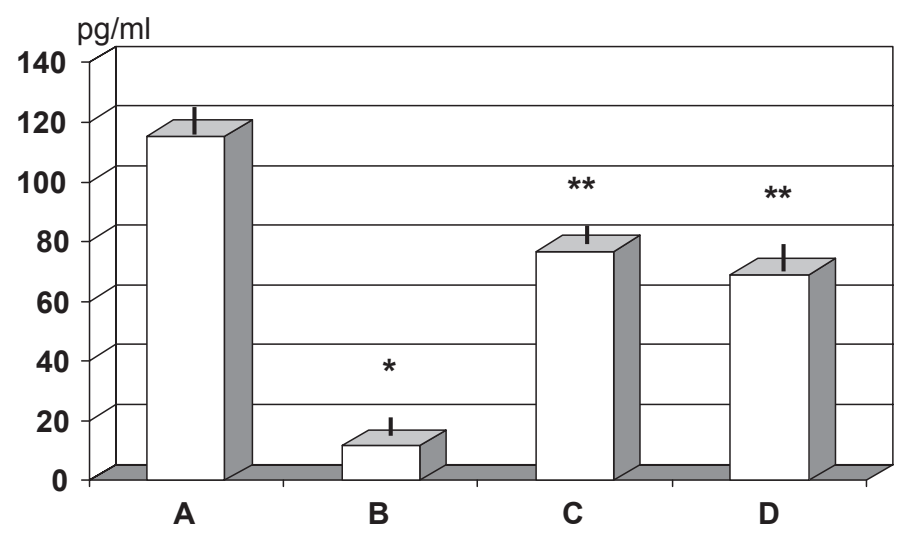

B

Uterus weight

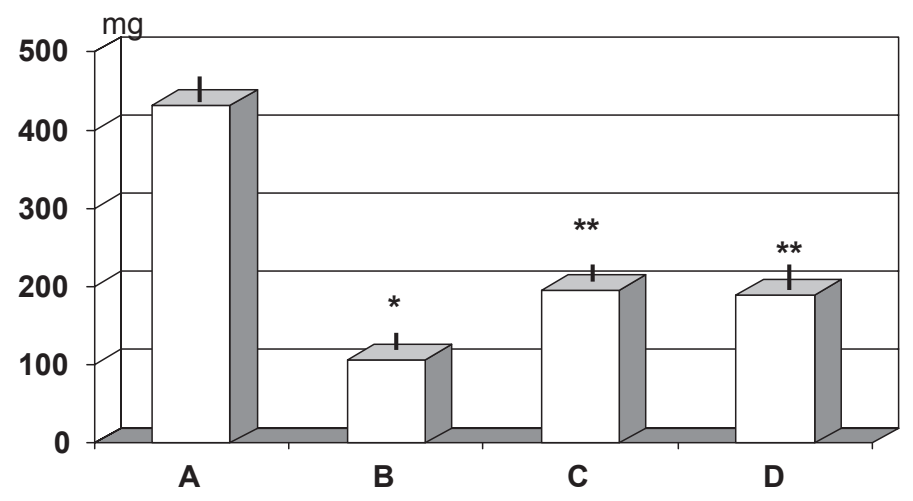

C

Osteocalcin level

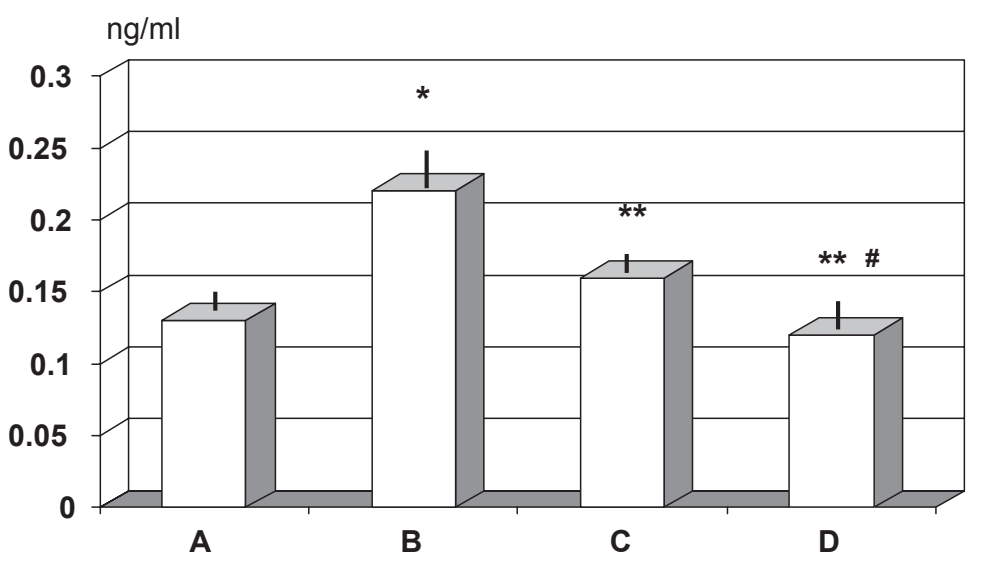

Figure I Effect of ovariectomy and supplementation of RCE and RCE + BP on estradiol A) uterine weight $\mathbf{B}$ ) and osteocalcin level C) (mean \pm SD).

Notes: *p $<0.01$ vs sham-operated (group A); ${ }^{* *} p<0.05$ vs untreated ovariectomy rats (group B), ${ }^{*} \mathrm{p}<0.05$ vs group $C$ (RCE-treated OVX rats).

Abbreviations: BP, calcium bicarbonate, sodium bicarbonate, magnesium and potassium carbonate, and bisodium phosphate; OVX, ovariectomized; RCE, red clover extract. 
A

Bone mineral density

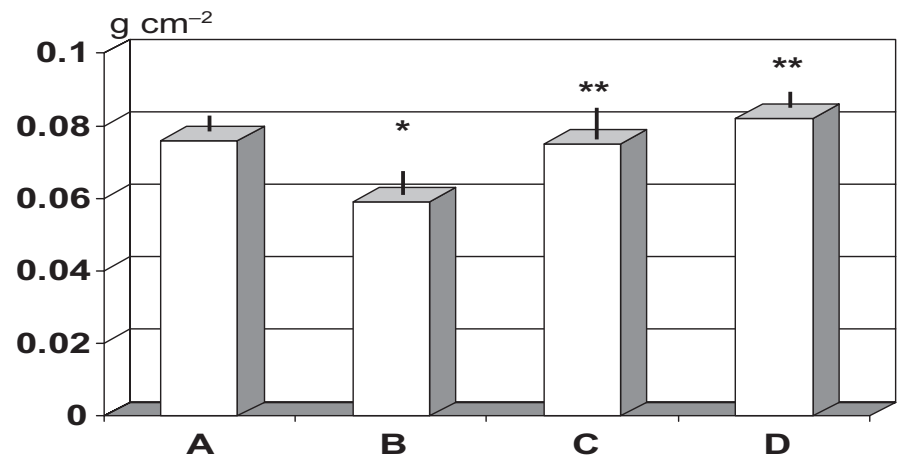

B

Bone mineral content

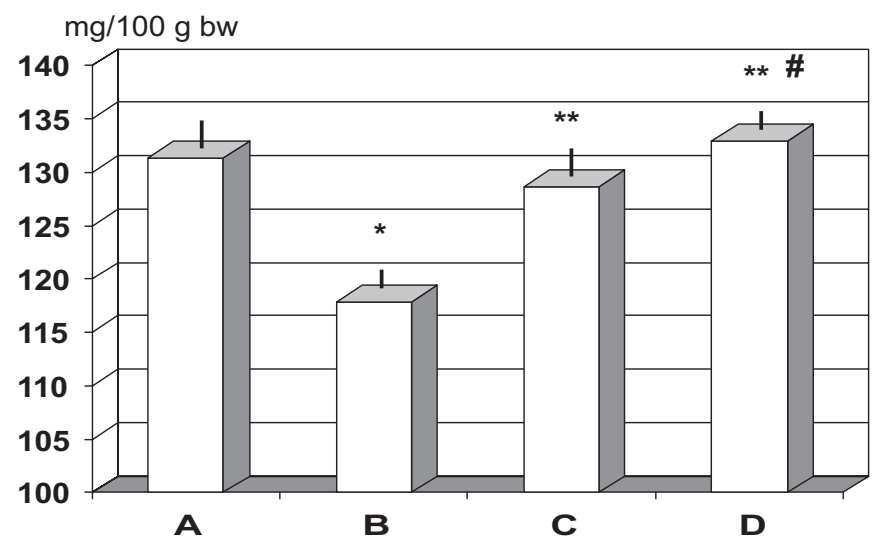

C

Percentage of cancellous bone mass in the femoral neck

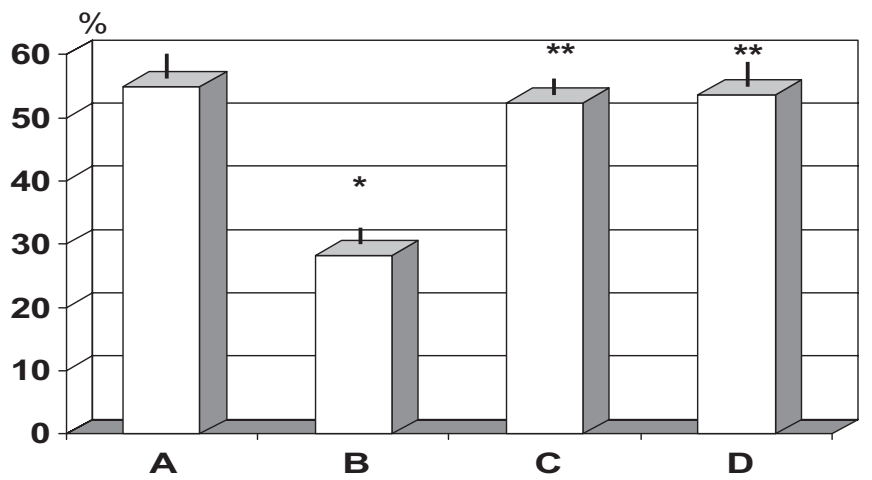

Figure 2 Effect of ovariectomy and supplementation of RCE and RCE + BP on bone mineral density A) bone mineral content/body wt ratio B) and cancellous bone mass C) (mean \pm SD).

Notes: ${ }^{*} \mathrm{p}<0.01$ vs sham-operated (group A); ${ }^{* *} \mathrm{p}<0.05$ vs untreated ovariectomy rats (group B); ${ }^{*} \mathrm{p}<0.05$ vs group $\mathrm{C}$ (RCE-treated OVX rats).

Abbreviations: BP, calcium bicarbonate, sodium bicarbonate, magnesium and potassium carbonate, and bisodium phosphate; $O V X$, ovariectomized; RCE, red clover extract. 


\section{Measurement of bone mechanics}

Mechanical testing of femoral necks showed that ovariectomized rats, whether specially supplemented or not, did not cause any significant change in energy absorption and stiffness, although RCE + BP-treated rats showed a not significant trend improvement as compared to group B. Moreover, no change among groups appeared in the deformation or breaking parameters measured by three point bending tests (data not shown). On the other hand untreated ovariectomy (group B) brought about a significant decrease of the maximal load of the femoral neck when compared to the sham rats ( $<<0.01$, Figure 3). Either treatment of RCE (group C) or $\mathrm{RCE}+\mathrm{BP}$ (group D) proved to be effective in totally preserving such mechanical failure. No significant difference appeared between these two therapeutic regimens.

\section{Effects of RCE or RCE + BP and ovariectomy on serum TALP, BALP, and urinary DPD}

As expected OVX rats showed a significant decrease of TALP $(\mathrm{p}<0.05)$ but no statistically significant difference appeared among the two different treatment groups (data not shown). Ovariectomy determined an over $80 \%$ increase of BALP level $(p<0.01$, Figure 4$)$ but both RCE and RCE + BP treatment mitigated such value to $15 \%-35 \%$ increase $(p<0.01$, Figure 4$)$. Further, the BALP decrease yielded by the combined RCE $+\mathrm{BP}$ treatment (group D) was about $16 \%$ lower than RCE alone (group C) and this was statistically significant $(p<0.05)$. Moreover, untreated OVX rats (group B) showed a remarkable increase of over $58 \%$ of urinary excretion of DPD (group B vs A, p < 0.05) while such parameter was within normal limits in both treatments groups ( $p<0.05$ vs group B).

\section{Discussion}

Osteoporosis is the most common metabolic bone disease associated with disharmonic bone remodeling due to decreased bone formation or accelerated bone resorption. Its regulators are changed by age, ions, hormones and growth factors. ${ }^{33-35}$ In the western world, the prevalence of osteoporosis and osteopenia in those aged 55 to 64 years is $20 \%$ and $37 \%$, respectively. This represents now a major public health threat, and its prevalence is expected to rise dramatically in the coming decades. ${ }^{36,37}$ Estrogens play an important role in skeletal homeostasis, and ovarian hormone deficiency is one of the most important risk factors for osteoporosis. Bone loss is most rapid in the first few years after menopause but it may persist into the postmenopausal years. Because of their selective estrogenic like activity, soy and red clover have been hypothesized to have a positive effect on bone mineral density as women age. There are clear bone-related benefits of hormone replacement therapy, but given the uncertain popularity of this approach there is a clinical need for alternative well tolerated pharmacological or nutritional treatments that can safely be used soon after the menopause and which effectively prevent bone loss and the development of osteoporosis. Phytoestrogens such as those derived from red clover may help with hot flushes, which are a common menopausal complaint. Red clover isoflavones have more

\section{Maximal load (N) of the femoral neck}

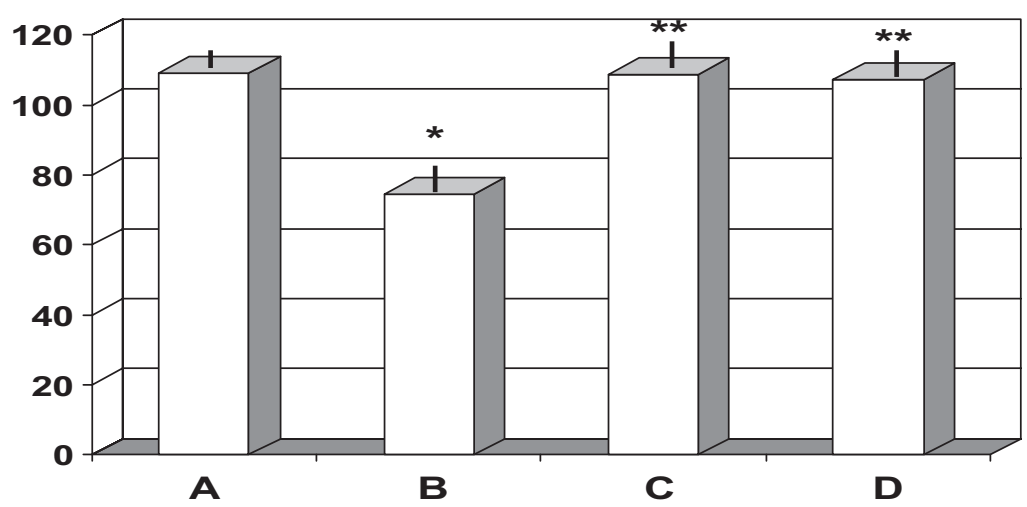

Figure 3 Effect of ovariectomy and supplementation of RCE and RCE + BP on maximal load on femoral neck (mean \pm SD)

Notes: ${ }^{*} \mathrm{p}<0.01$ vs sham-operated (group A); ${ }^{* *} \mathrm{p}<0.05$ vs untreated ovariectomy rats (group B).

Abbreviations: $\mathrm{BP}$, calcium bicarbonate, sodium bicarbonate, magnesium and potassium carbonate, and bisodium phosphate; OVX, ovariectomized; RCE, red clover extract. 


\section{Urinary DPD}

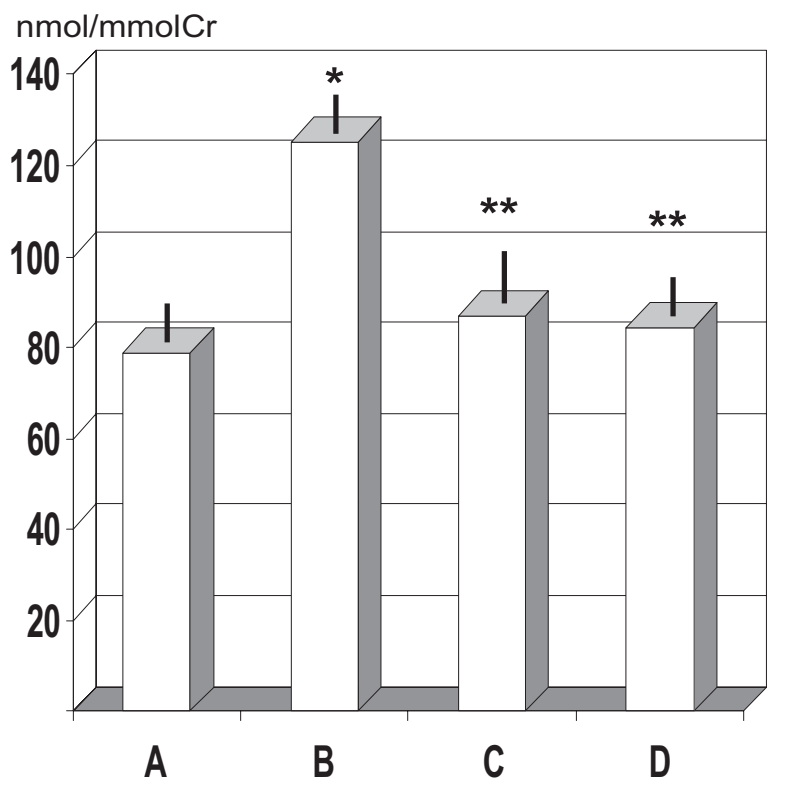

\section{Bone-specific ALP}

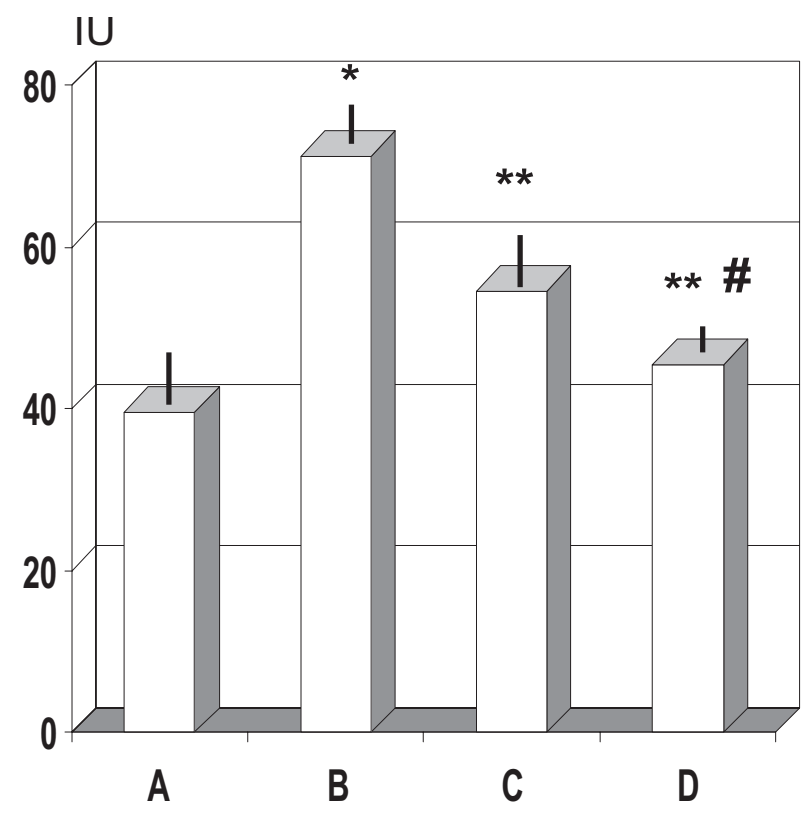

Figure 4 Effect of ovariectomy and supplementation of RCE and RCE + BP on urinary DPD excretion and bone-specific ALP.

Notes: ${ }^{*} p<0.01$ vs sham-operated (group A); ${ }^{* *} p<0.05$ vs untreated ovariectomy rats (group $B$ ); ${ }^{*} p<0.05$ vs group $C$ (RCE-treated OVX rats).

Abbreviations: ALP, alkaline phosphatase; BP, calcium bicarbonate, sodium bicarbonate, magnesium and potassium carbonate, and bisodium phosphate; OVX, ovariectomized; RCE, red clover extract.

evidence-based studies than most herbal medicinal products, although the literature is beset by difference in methodologies making direct comparison between them difficult. In the present study we employed dosages of red clover which are amenable to clinical practice application and found that such supplementation proved to be effective in increasing the BMD and BMC together with counteracting the drastic decrease of cancellous bone mass due to OVX.

It has been reported that both bone resorption and bone formation are promoted by ovariectomy. ${ }^{38}$ Estrogen induces a decrease in bone resorption and may also have some anabolic effect, particularly in high doses in postmenopausal women at all ages..$^{39,40}$ Treatment with RCE showed to significantly mitigate the post-OVX fall of estradiol level which partly maintained their trophic effect also on the uterine tissue. It is well known that the body's acid-base balance plays an important role in bone health and is modifiable by diet. ${ }^{41,42}$ Indeed, the organic acids which are produced during metabolism and the hepatic oxidation of sulfur-amino acids lower blood $\mathrm{pH}$ through increased production of hydrogen ions..$^{43}$ We have recently shown that, by employing the same modified alkaline supplementation used in this study, it could prevent the chronic acidosisinduced BMD loss with increased urinary output of DPD, calcium, and phosphorus in male rats fed an acidogenic high-protein diet. ${ }^{25}$ In daily life, the alkaline dietary components such as potassium, calcium, and magnesium act as physiological buffers for organic acids. However, if insufficient levels of buffering capacity are available from alkaline salts, mild metabolic acidosis may subtly ensue even in healthy people, especially women ${ }^{44}$ with all the related bone derangement on the long run..$^{42,45,56}$ In the present study, the addition of BP to RCE proved to further improve bone turnover markers such as urinary excretion of $\mathrm{DPD}, \mathrm{BALP},{ }^{47}$ and serum osteocalcin. Osteocalcin is unique to bone tissue and it is the most abundant in noncollagenous bone protein. It is formed in the osteoblast and deposited in bone matrix although a small fraction of the newly synthesized component is released into the bloodstream while its activity and synthesis increase when BMD is decreased due to higher bone resorption. ${ }^{48}$ In agreement with what was recently reported by Occhiuto and colleagues, ${ }^{49}$ who used larger dosages of red clover isoflavones, the mechanical testing showed a significant preservation of maximal load applied to the femoral neck in rats treated with RCE although either energy, stiffness, and deformation were not affected by OVX nor further improved by treatment. Different dosage and longer observation time might be factors to be taken into account in further studies when assessing such specific variables. 
Although the ovariectomized rat is widely used as a model of postmenopausal osteoporosis a number of cautions have to be considered when assessing its relevance in clinics, ${ }^{50}$ genetic differences with humans included. Nonetheless, the present finding show that RCE preparation employed at dosages not far from the ones applied in clinical practice are effective in reducing ovariectomy-induced bone loss by reducing bone turnover via inhibition of bone resorption and this effects might be further enhanced by a mild alkalinization. Specific supplemental administration rather than dietary implementation seems to be a more defined approach with therapeutic modality without any reported negative effect while experimental studies are awaited in male osteoporosis given the increasing interest in estrogen receptor modulators also in male osteopenia.

\section{Disclosure}

There was no funding of the research nor ties with any firm involved in red clover and no conflict of interest exists. The research costs were handled thanks to an unrestricted grant from Canova Foundation, Italy, a nonprofit organization devoted to research.

\section{References}

1. Cheung AM, Feig DS, Kapral M, Diaz-Cranados N, Dodin S. Canadian Task Force on Preventive Health Care. Prevention of osteoporosis and osteoporotic fractures in postmenopausal women: Recommendation statement from the Canadian Task Farce on Preventive Health Care. CMAJ. 2004;170:1665-1667.

2. Consensus Development Conference. Diagnosis, prophylaxis and treatment of osteoporosis. Am J Med. 1993;94:646-650.

3. Kanis JA, McCloskey EV, Johansson H, Strom O, Borgstrom F, Oden A; National Osteoporosis Guideline Group. Case finding for the management of osteoporosis with FRAX-assessment and intervention thresholds for the UK. Osteoporos Int. 2008;19:1395-1408.

4. Management of Postmenopausal Osteoporosis: Position Statement of the North American Menopause Society. Menopause. 2002;9:84-101.

5. Rossouw JE, Anderson GL, Prentice RL, LaCroix AZ, Kooperberg C, Stefanick ML. Risks and benefits of estrogen plus progestin in healthy postmenopausal women: Principal results from the Women's Health Initiatíve randomized controlled trial. JAMA. 2002;288:321-323.

6. Chen YM, Ho SC, Lam SS, Ho SS, Woo JL. Soy isoflavones have a favorable effect on bone loss in Chinese postmenopausal women with lower bone mass: a double-blind, randomized, controlled trial. J Clin Endocrinol Metab. 2003;88:4740-4747.

7. Adlercreutz H, Mazur M. Phyto-oestrogens and westem dìseases. Ann Med. 1997;29:95-120.

8. de Kleijn MJJ, van der Schouw YT, Wilson PW, et al. Intake of dietary phytoestrogens is low in postmenopausal women in the United States: the Framingham study (1-4). J Nutr. 2001;131:1826-1832.

9. Brynin R. Soy and its isoflavones: a review of their effects on bone density. Altern Med Rev. 2002;7:317-327.

10. Mazur W, Adlercreutz H. Overview of naturally occurring endocrine active substances in the human diet in relation to human health Nutrition. 2000;16:654-658.

11. Whitten PL, Patisaul HB. Cross-species and interassay comparisons of phytoestrogen actian. Environ Health Perspect. 2001;109(Suppl 1):5-20.
12. Arjmandi BH, Alekel L, Hollis BW, Amin D, Stacewicz-Sapuntzakis M, Guo P. Kukreja SC. Dietary soybean protein prevents bone loss in an ovariectomized rat model of osteoporosis. J Nutr. 1996;126:161-167.

13. Adlercreutz H. Western diet and western disease: some hormonal and biochemical mechanisms and associations. Scand J Clin Lab Invest. 1990;50(Supp 1201):3-23.

14. Occhiuto F, Zangla G, Samperi S, et al. The phytoestrogenic isoflavones from Trifolium pratense L. (Red clover) protects human cortical neurons from glutamate toxicity. Phytomedicine. 2008;15:676-682.

15. Umland EM. Treatment strategies for reducing the burden of menopause-associated vasomotor symptoms. J Manag Care Pharm. 2008;14(3 Suppl):14-19.

16. Simoncini T, Garibaldi S, Fu XD, Pisaneschi S, Begliuomini S, Baldacci C, Lenzi E, Goglia L, Giretti MS, Genazzani AR. Effects of phytoestrogens derived from red clover on atherogenic adhesion molecules in human endothelial cells. Menopause. 2008;15:542-550.

17. Powles TJ, Howell A, Evans DG, et al. Red clover isoflavones are safe and well tolerated in women with a family history of breast cancer. Menopause Int. 2008;14:6-12.

18. Clifton-Bligh PB, Baber RJ, Fulcher GR, Nery ML, Moreton T. The effect of isoflavones extracted from red clover (Rimostil) on lipid and bone metabolism. Menopause. 2001;8:259-265.

19. Atkinson C, Compston JE, Day NE, Dowsett M, Bingham SA. The effects of phytoestrogen isoflavones on bone density in women: a double-blind, randomized, placebo-controlled trial. Am J Clin Nutr. 2004;79:326-333.

20. Miller S. Models of skeletal osteopenia in the rat. J Histotechnol. 1997;20:209-213.

21. Dai R, Ma Y, Sheng Z, et al. Effects of genistein on vertebral trabecular bone microstructure, bone mineral density, microcracks, osteocyte density, and bone strength in ovariectomized rats. J Bone Miner Metab. 2008;26:342-349.

22. Lemann J Jr, Bushinsky DA, Hamm LL. Bone buffering of acid and base in humans. Am J Physiol Renal Physiol. 2003;285:F811-832.

23. Sebastian A, Harris ST, Ottaway JH, Todd KM, Morris RC Jr. Improved mineral balance and skeletal metabolism in postmenopausal women treated with potassium bicarbonate. $N$ Engl J Med. 1994;330:1776-1781.

24. Marangella M, Di Stefano M, Casalis S, Berutti S, D’Amelio P, Isaia GC. Effects of potassium citrate supplementation on bone metabolism. Calcif Tissue Int. 2004;74:330-335.

25. Chui DH, Marotta F, Liu T, et al. Effect of modified alkaline supplementation on bone metabolic turnover in rats. J Biol Regul Homeost Agents. 2008;22:225-231.

26. Marotta F, Chui de H, Lorenzetti A, Fayet F, Liu T, Marandola P. Muscular metabolism in aged rats under exhaustive exercise: effect of a modified alkaline supplementation. Rejuvenation Res. 2008;11:519-522.

27. Brandao-Burch A, Utting JC, Orriss IR, Arnett TR. Acidosis inhibits bone formation by osteoblasts in vitro by preventing mineralization. Calcif Tissue Int. 2005;77:167-174.

28. Arnett T. Regulation of bone cell function by acid-base balance. Proc Nutr Soc. 2003;62:511-520.

29. Bushinsky DA, Parker WR, Alexander KM, Krieger NS. Metabolic, but not respiratory, acidosis increases bone PGE(2) levels and calcium release. Am J Physiol Renal Physiol. 2001;281:F1058-F1066.

30. Krieger NS, Bushinsky DA, Frick KK. Cellular mechanisms of bone resorption induced by metabolic acidosis. Semin Dial. 2003; 16:463-466.

31. Gundberg CM, Clough ME, Carpenter TO. Development and validation of a radioimmunoassay for mouse osteocalcin: paradoxical response in the Hyp mouse. Endocrinology. 1992;130:1909-1915.

32. Matsumoto J, Kobayashi S, Kikuchi S, Araki T. Femoral bone density and changes therein associated with differing histories of pregnancy and lactation in aged rats. J Nippon Med Sch. 2000;67:18-23.

33. Baldock PA, Morris HA, Need AG, Moore RJ, Durbridge TC. Variation in the short-term changes in bone cell activity in three regions of the distal femur immediately following ovariectomy. J Bone Miner Res. 1998;13:1451-1457. 
34. Seeman E. Structural basis of growth-related gain and age-related loss of bone strength. Rheumatology (Oxford). 2008;47(Suppl 4):iv2-8.

35. Stanworth RD, Jones TH. Testosterone for the aging male; current evidence and recommended practice. Clin Interv Aging. 2008;3:25-44.

36. Sharma S, Tandon VR, Mahajan A, Kour A, Kumar D. Preliminary screening of osteoporosis and osteopenia in urban women from Jammu using calcaneal QUS. Indian J Med Sci. 2006;60:183-189.

37. Anastasopoulou C, Rude RK. Bone mineral density screening: assessment of influence on prevention and treatment of osteoporosis. Endocr Pract. 2002;8:199-201.

38. Wronski TJ, Yen CF. The ovariectomized rat as an animal model for postmenopausal bone loss. Cells Mater. 1991;(Suppl 1):69-74.

39. Prestwood KM, Pilbeam CC, Burleson JA, Woodiel FN, Delmas PD, Deftos LI. The short-term effects of conjugated estrogen on bone turnover in older women. J Clin Endocrinol Metab. 1994;79:366-371.

40. Vedi S, Purdie DVV, Ballard P, Bord S, Cooper AC, Compston SE. Bone remodeling and structure in postmenopausal women treated with longterm, high-dose estrogen therapy. Osteoporosis Int. 1999;10:52-58.

41. New SA. Nutrition Society Medal lecture. The role of the skeleton in acid-base homeostasis. Proc Nutr Soc. 2002;61:151-164.

42. Buclin T, Cosma M, Appenzeller M, et al. Diet acids and alkalis influence calcium retention in bone. Osteoporos Int. 2001;12:493-499.

43. Remer T. Influence of diet on acid-base balance. Semin Dial. 2000;13:221-226.
44. Welch AA, Bingham SA, Reeve J, Khaw KT. More acidic dietary acid-base load is associated with reduced calcaneal broadband ultrasound attenuation in women but not in men: results from the EPICNorfolk cohort study. Am J Clin Nutr. 2007;85:1134-1141.

45. Frassetto LA, Todd KM, Morris RC Jr, Sebastian A. Estimation of net endogenous noncarbonic acid production in humans from diet potassium and protein contents. Am J Clin Nutr. 1998;68:576-583.

46. Lemann J Jr, Bushinsky DA, Hamm LL. Bone buffering of acid and base in humans. Am J Physiol Renal Physiol. 2003;285:F811-F832.

47. Van Straalen JP, Sanders E, Prummel MF, Sanders GT. Bone-alkaline phosphatase as indicator of bone formation. Clin Chim Acta. 1991;201:27-33.

48. Kumm J, Ivaska KK, Rohtla K, Vaananen K, Tamm A. Urinary osteocalcin and other markers of bone metabolism: the effect of risedronate therapy. Scand J Clin Lab Invest. 2008;11:1-5.

49. Occhiuto F, Pasquale RD, Guglielmo G, et al. Effects of phytoestrogenic isoflavones from red clover (Trifolium pratense L.) on experimental osteoporosis. Phytother Res. 2007;21:130-134.

50. Namkung-Matthai H, Appleyard R, Jansan J, Hao Lin J, Maastricht S, Swain M. Osteoporosis influences the early period of fracture healing in a rat osteoporotic model. Bone. 2001;28:80-86. 